\title{
UNIQUE SOLVABILITY OF A DIRICHLET PROBLEM FOR A FRACTIONAL PARABOLIC EQUATION USING ENERGY-INEQUALITY METHOD
}

\author{
BENAOUA ANTARA ${ }^{1,2}$, OUSSAEIF TAKI-EDDINE ${ }^{1,2}$, AND REZZOUG IMAD $^{1,2}$
}

\begin{abstract}
In this paper, we establish sufficient conditions for the existence and uniqueness of the solution in fractional functional space for a class of initial boundaryvalue problems for a class of partial fractional parabolic differential equations that include a fractional derivative of Caputo. The results are established by the application of the method based on a priori estimate "energy inequality" and the density of the range of the operator generated by the problem considered.
\end{abstract}

\begin{abstract}
Встановлені достатні умови існування та єдиності розв'язку з дробового функціонального простору для одного класу початково-крайових задач для деяких дробово-параболічних диференціальних рівнянь із дробовою похідною Капуто. Результати отримано шляхом застосування методу енергетичних нерівностей. Доведена щільність образу оператора, що відповідає задачі.
\end{abstract}

\section{INTRODUCTION}

Fractional differential equations (FDEs) are obtained by generalizing differential equations to an arbitrary order. Since fractional differential equations are used to model complex phenomena, they play a crucial role in engineering, physics and applied mathematics. Therefore they have been generating increasing interest from engineers and scientist in recent years. Since FDEs have memory, nonlocal relations in space and time, complex phenomena can be modeled by using these equations. Due to this fact, materials with memory and hereditary effects, through strongly anomalous media. Indeed, we can find numerous applications in viscoelasticity, electro-chemistry, signal processing, control theory, porous media, fluid flow, rheology, diffusive transport, electrical networks, electromagnetic theory and probability, signal processing, and many other physical processes are diverse applications of FDEs $[1-7]$.

Recently, there has been a significant development in fractional differential and partial differential equations; see the monographs of Kilbas et al. [8], Miller and Ross [9], Samko et al. [10] and the papers of Agarwal et al. [11], Anguraj A. and Karthikeyan P. [12], Belmekki et al. [13], Daftardar-Gejji and Jafari [18], Furati and Tatar [25,26], Kaufmann and Mboumi [27], Kilbas and Marzan [28], Yu and Gao [32], Oussaeif [33], and also the general references in Baleanu et al. [34], and the references therein. However, many phenomena can better be described by integral boundary conditions. Integral boundary conditions are encountered in various applications such as population dynamics, blood flow models, chemical engineering and cellular systems.

The study of existence and uniqueness, periodicity, asymptotic behavior, stability, and methods of analytic and numerical solutions of fractional differential equations have been studied extensively in a large cycle works. But there are not many works in the fractional field of partial differential equation, this is due to the difficulty of applying classical theories and methods to a field of farctional partial differential eqation. Motivated by this, we conducted a detailed and thorough study in this field to see the behavior of the solution to these problems using the classic energy estimat method. Then, the present

Keywords. Partial fractional differential equation, energy inequality; existence; uniqueness. 
paper is devoted to the study of initial-boundary value problem for a parabolic equation with time-fractional derivative with Dirichlet condtion, which has not been studied so far.

\section{Preliminaries and FUnCtional spaces}

Let $\Omega=[0, T]$ be a finite interval of the real numbers $\mathbb{R}$ and $\boldsymbol{\Gamma}(\cdot)$ denote the gamma function. For any positive integer $0<\alpha<1$, the Caputo derivative are the Riemann Liouville derivative are, respectively, defined as follows:Let $\Gamma$ (.) denote the gamma function. For any positive integer $0<\alpha<1$, the Caputo derivative are the Riemann Liouville derivative are, respectively, defined as follows:

(1) The left Caputo derivatives:

$$
{ }^{C} D_{t}^{\alpha} u(x, t):=\frac{1}{\Gamma(1-\alpha)} \int_{0}^{t} \frac{\partial u(x, \tau)}{\partial \tau} \frac{1}{(t-\tau)^{\alpha}} d \tau .
$$

(2) The left Riemann-Liouville derivatives:

$$
{ }^{R} D_{t}^{\alpha} u(x, t):=\frac{1}{\Gamma(1-\alpha)} \frac{\partial}{\partial t} \int_{0}^{t} \frac{u(x, \tau)}{(t-\tau)^{\alpha}} d \tau .
$$

(3) The right Caputo derivatives:

$$
{ }_{t}^{C} D^{\alpha} u(x, t):=\frac{-1}{\Gamma(1-\alpha)} \int_{t}^{T} \frac{\partial u(x, \tau)}{\partial \tau} \frac{1}{(\tau-t)^{\alpha}} d \tau .
$$

(4) The right Riemann-Liouville derivatives:

$$
{ }_{t}^{R} D^{\alpha} u(x, t)=\frac{-1}{\Gamma(1-\alpha)} \frac{\partial}{\partial t} \int_{t}^{T} \frac{u(x, \tau)}{(\tau-t)^{\alpha}} d \tau,
$$

Many authors think that the Caputo's version is more natural because it allows the handling of inhomogeneous initial conditions in a easier way. Then the two definitions (2.1) and (2.2) are linked by the following relationship, which can be verified by a direct calculation:

$$
{ }^{R} D_{t}^{\alpha} u(x, t)={ }^{C} D_{t}^{\alpha} u(x, t)+\frac{u(x, 0)}{\Gamma(1-\alpha) t^{\alpha}} .
$$

Definition 2.1. [37] For any real $\sigma>0$ and finite interval [a, b] of the real axis $\mathrm{R}$, we define the semi-norm:

$$
|u|_{l_{H^{\sigma}(\Omega)}}^{2}:=\left\|{ }^{R} D_{t}^{\sigma} u\right\|_{L_{2}(\Omega)}^{2}
$$

and norm:

$$
\|u\|_{l_{H^{\sigma}}(\Omega)}:=\left(\|u\|_{L_{2}(\Omega)}^{2}+|u|_{l_{H^{\sigma}}(\Omega)}^{2}\right)^{\frac{1}{2}},
$$

we then define ${ }^{l} H_{0}^{\sigma}(\Omega)$ as the closure of $C_{0}^{\infty}(\Omega)$ with respect to the norm $\|\cdot\|_{l} H^{\sigma}(\Omega)$.

Definition 2.2. [37] For any real $\sigma>0$, we define the semi-norm:

$$
|u|_{r_{H^{\sigma}(\Omega)}}^{2}:=\left\|{ }_{t}^{R} D^{\sigma} u\right\|_{L_{2}(\Omega)}^{2}
$$

and norm:

$$
\|u\|_{r_{H^{\sigma}(\Omega)}}:=\left(\|u\|_{L_{2}(\Omega)}^{2}+|u|_{r_{H^{\sigma}}(\Omega)}^{2}\right)^{\frac{1}{2}},
$$

we then define ${ }^{R} H_{0}^{\sigma}(\Omega)$ as the closure of $C_{0}^{\infty}(\Omega)$ with respect to the norm $\|\cdot\|_{r} H^{\sigma}(\Omega)$. 
Definition 2.3. For any real $\sigma>0$, we define the semi-norm:

$$
|u|_{c_{H^{\sigma}(\Omega)}}=\left|\frac{\left({ }^{R} D_{t}^{\sigma} u,{ }_{t}^{R} D^{\sigma} u\right)_{L^{2}(\Omega)}}{\cos (\sigma \pi)}\right|^{1 / 2}
$$

and norm:

$$
\|u\|_{c_{H^{\sigma}(\Omega)}}=\left(\|u\|_{L^{2}(\Omega)}^{2}+|u|_{c_{H^{\sigma}}(\Omega)}^{2}\right)^{1 / 2} .
$$

Lemma 2.4. $[36,37]$ For any real $\sigma \in \mathbb{R}_{+}$, if $u \in{ }^{l} H^{\alpha}(\Omega)$ and $v \in C_{0}^{\infty}(\Omega)$, then

$$
\left({ }^{R} D_{t}^{\sigma} u(t), v(t)\right)_{L^{2}(\Omega)}=\left(u(t),{ }_{t}^{R} D^{\sigma} v(t)\right)_{L^{2}(\Omega)} .
$$

Lemma 2.5. [36, 37] For $0<\sigma<2, \sigma \neq 1, u \in H_{0}^{\frac{\sigma}{2}}(\Omega)$, on a :

$$
{ }^{R} D_{t}^{\sigma} u(t)={ }^{R} D_{t}^{\frac{\sigma}{2}} R D_{t}^{\frac{\sigma}{2}} u(t) .
$$

Lemma 2.6. $[36,37]$ For $\sigma \in \mathbb{R}_{+}, \sigma \neq n+\frac{1}{2}$, the semi- norms $|\cdot|_{l_{H^{\sigma}(\Omega)},}|\cdot|_{r_{H^{\sigma}}(\Omega)}$ and $|\cdot|_{C_{H} \sigma(\Omega)}$ are equivalent. Then we pose

$$
|\cdot|_{H_{H^{\sigma}(\Omega)}} \cong|\cdot|_{r_{H^{\sigma}(\Omega)}} \cong|\cdot|_{c_{H^{\sigma}(\Omega)}} .
$$

Lemma 2.7. $[36,37]$ For any real $\sigma>0$, the space ${ }^{R} H_{0}^{\sigma}(\Omega)$ with respect to the norm (2.5) is complete.

Definition 2.8. We denote by $L_{2}\left(0, T, L_{2}(0,1)\right):=L_{2}(Q)$ the space of functions which are square integrable in the Bochner sense, with the scalar product

$$
(u, w)_{L_{2}\left(0, T, L_{2}(0,1)\right)}=\int_{0}^{T}((u, \cdot),(w, \cdot))_{L_{2}(0,1)} d t .
$$

Since the space $L_{2}(0,1)$ is a Hilbert space, it can be shown that $L_{2}\left(0, T, L_{2}(0,1)\right)$ is a Hilbert space as well. Let $C^{\infty}(0, T)$ denote th space of infinitely diffrentiable functions on $(0, T)$ and $C_{0}^{\infty}(0, T)$ denote th space of infinitely diffrentiable functions with compact support in $(0, T)$.

\section{Statement of problem}

In the rectangular domain $Q=(0, d) \times(0, T)$, with $d, T<\infty$ and $0<\alpha<1$, we shall study the existence and uniqueness of solutions $u=u(x, t)$ to the following fractional parabolic problem :

$$
\begin{cases}{ }^{C} D_{t}^{\alpha} u(x, t)-\frac{\partial}{\partial x}\left(a(x, t) \frac{\partial u(x, t)}{\partial x}\right)+b(x, t) u(x, t)=\widetilde{f}(x, t) & \text { in } Q ; \\ u(x, 0)=\varphi(x) & \forall x \in(0, d), \\ u(0, t)=u(d, t)=0 & \forall t \in(0, T) .\end{cases}
$$

We consider the following fractional parabolic equation of the type:

$$
\mathcal{L} u={ }^{C} D_{t}^{\alpha} u-\frac{\partial}{\partial x}\left(a \frac{\partial u}{\partial x}\right)+b u=\widetilde{f},
$$

with the initial condition :

$$
\ell u=u(x, 0)=\varphi(x), \quad \forall x \in(0, d),
$$

and Dirichlet condition :

$$
u(0, t)=u(d, t)=0, \quad \forall t \in(0, T) .
$$

Where $a, b, \tilde{f}$ and $\varphi$ are known functions.

We shall assume that the function $\varphi$ satisfies a compatibility conditions, i.e.,

$$
\varphi(0)=\varphi(d)=0 .
$$


And the functions $a, b$ verify:

$$
\begin{aligned}
& 0<a_{0} \leq a(x, t) \leq a_{1}, \\
& 0<b_{0} \leq b(x, t) \leq b_{1} .
\end{aligned}
$$

Now, we shall introduce a new function :

$$
v(x, t)=u(x, t)-U(x) \Longrightarrow u(x, t)=v(x, t)+U(x),
$$

where

So, we get

$$
\varphi(x)=U(x) .
$$

$$
\left\{\begin{array}{l}
{ }^{C} D_{t}^{\alpha} v(x, t)-\frac{\partial}{\partial x}\left(a(x, t) \frac{\partial v(x, t)}{\partial x}\right)+b(x, t) v(x, t)=\widetilde{f}(x, t)-\mathcal{L} \varphi(x)=f(x, t) \text { in } Q, \\
v(x, 0)=0 \quad \forall x \in(0, d) \\
v(0, t)=v(d, t)=0 \quad \forall t \in(0, T)
\end{array}\right.
$$

Such that

$$
{ }^{C} D_{t}^{\alpha} v(x, t)-\frac{\partial}{\partial x}\left(a(x, t) \frac{\partial v(x, t)}{\partial x}\right)+b(x, t) v(x, t)=f(x, t)
$$

with the initial condition

$$
\ell v=v(x, 0)=0, \quad \forall x \in(0, d)
$$

the boundary condition of Dirichlet type

$$
v(0, t)=v(d, t)=0, \quad \forall t \in(0, T)
$$

where

$$
f(x, t)=\widetilde{f}(x, t)+\frac{\partial}{\partial x}\left(a(x, t) \frac{\partial \varphi(x)}{\partial x}\right)-b(x, t) \varphi(x) .
$$

\section{Estimation À PRIORI}

The method used here is one of the most efficient functional analysis methods and important techniques for solving partial differential equations with integral conditions, which has been successfully used in investigating the existence, uniqueness, and continuous dependence of the solutions of PDE's, the so-called a priori estimate method or the energy-inequality method. This method is essentially based on the construction of multiplicators for each specific given problem, which provides the a priori estimate from which it is possible to establish the solvability of the posed problem. More precisely, the proof is based on an energy inequality and the density of the range of the operator generated by the abstract formulation of the stated problem, so to investigated the posed problem, we introduce the needed function spaces. In this paper, we prove the existence and the uniqueness for solution of the problem (3.1) - (3.3) as a solution of the operator equation

$$
L v=\mathcal{F},
$$

Where $L=(\mathcal{L}, \ell)$, with domain of difinition $B$ consisting of functions $v \in L^{2}(Q)$, such that $v,{ }^{C} D_{t}^{\alpha} v, \frac{\partial v}{\partial x} \in L^{2}(Q)$ and $v$ satisfies the condition (3.3).

The operator $L$ is considered from $B$ to $F$, where $B$ is the Banach space consisting of all functions $v(x, t)$ having a finite norm

$$
\|v\|_{B}^{2}=\left\|{ }^{C} D_{t}^{\frac{\alpha}{2}} v\right\|_{L^{2}(Q)}^{2}+\|v\|_{L^{2}(Q)}^{2}+\left\|\frac{\partial v}{\partial x}\right\|_{L^{2}(Q)}^{2},
$$

and $F$ is the Hilbert space consisting of all elements $\mathcal{F}=(f, 0)$ for which the norm $L^{2}(Q)$ is finite. 
Theorem 4.1. For any function $u \in B$, we have the inequality

$$
\|v\|_{B} \leq k\|L v\|_{L^{2}(Q)}
$$

where $k$ is a positive constant independent of $v$.

Proof. Multiplying the equation (3.1) by the following function:

$$
M v=v(x, t),
$$

and integrating over $Q=(0, d) \times(0, T)$, we get

$$
\begin{aligned}
& \int_{Q} \mathcal{L} v \cdot M v d x d t \\
&=\int_{Q}{ }^{C} D_{t}^{\alpha} v(x, t) \cdot v(x, t) d x d t-\int_{Q} \frac{\partial}{\partial x}\left(a(x, t) \frac{\partial v(x, t)}{\partial x}\right) v(x, t) d x d t \\
& \quad+\int_{Q} b(x, t) \cdot v^{2}(x, t) d x d t \\
& \quad=\int_{Q} f(x, t) \cdot v(x, t) d x d t .
\end{aligned}
$$

As $v(x, 0)=0$, we have ${ }^{C} D_{t}^{\alpha} v(x, t)={ }^{R} D_{t}^{\alpha} v(x, t)$, so by applying Lemmas 1,2 et 3 , we find

$$
\begin{aligned}
& \int_{Q}{ }^{C} D_{t}^{\alpha} v(x, t) \cdot v(x, t) d x d t \\
& =\left({ }^{C} D_{t}^{\alpha} v(x, t), v(x, t)\right)_{L^{2}(Q)} \\
& =\left({ }^{C} D_{t}^{\frac{\alpha}{2}}{ }^{C} D_{t}^{\frac{\alpha}{2}} v(x, t), v(x, t)\right)_{L^{2}(Q)} \quad \text { (According to Lemma 2) } \\
& =\left({ }^{C} D_{t}^{\frac{\alpha}{2}} v(x, t),{ }_{t}^{C} D^{\frac{\alpha}{2}} v(x, t)\right)_{L^{2}(Q) \quad \text { (According to Lemma 1) }} \\
& =\cos \left(\frac{\alpha}{2} \pi\right)|v|_{c^{H} H^{\frac{\alpha}{2}}}^{2}(Q) \quad \text { (According to Definition 3) }
\end{aligned}
$$

then, according to Lemma 3, we get that

$$
\begin{aligned}
\int_{Q}{ }^{C} D_{t}^{\alpha} v(x, t) \cdot v(x, t) d x d t=\cos \left(\frac{\alpha}{2} \pi\right)|v|_{c^{\frac{\alpha}{2}}}^{2}(Q) & \cong \cos \left(\frac{\alpha}{2} \pi\right)|v|_{l H^{\frac{\alpha}{2}}}^{2}(Q) \\
& =\cos \left(\frac{\alpha}{2} \pi\right)\left\|{ }^{C} D_{t}^{\frac{\alpha}{2}} v\right\|_{L^{2}(Q)}^{2} .
\end{aligned}
$$

By integration by parts over $(0, d)$ we get

$$
\begin{aligned}
-\int_{Q} \frac{\partial}{\partial x} & \left(a(x, t) \frac{\partial v(x, t)}{\partial x}\right) v(x, t) d x d t \\
& =-\int_{0}^{T} \int_{0}^{d} \frac{\partial}{\partial x}\left(a(x, t) \frac{\partial v(x, t)}{\partial x}\right) v(x, t) d x d t \\
& =-\left.\int_{0}^{T} a(x, t) \frac{\partial v(x, t)}{\partial x} v(x, t)\right|_{x=0} ^{x=d} d t+\int_{Q} a(x, t)\left(\frac{\partial v(x, t)}{\partial x}\right)^{2} d x d t \\
& =\int_{Q} a(x, t)\left(\frac{\partial v(x, t)}{\partial x}\right)^{2} d x d t .
\end{aligned}
$$

By using the Cauchy inequality with $\varepsilon$, for $\varepsilon<2 b_{0}$; and becaude of the equivalent of the semi-norms $|\cdot|_{l_{H^{\sigma}(Q)}}$ and $|\cdot|_{c H^{\sigma}(Q)}$, there is a positive constant $m$ such that

$$
m|\cdot|_{l_{H^{\sigma}}(Q)} \leqslant|\cdot|_{c_{H^{\sigma}}(Q)} \cdot
$$


So, we have

$$
m^{2}|\cdot|_{l_{H \sigma}(Q)}^{2} \leqslant|\cdot|_{c_{H^{\sigma}}(Q)}^{2}
$$

which gives that

$$
\begin{aligned}
\int_{Q}{ }^{C} D_{t}^{\alpha} v(x, t) \cdot v(x, t) d x d t=\cos \left(\frac{\alpha}{2} \pi\right)|v|_{c^{\frac{\alpha}{2}}(Q)}^{2} \geqslant & m^{2} \cos \left(\frac{\alpha}{2} \pi\right)|v|_{l_{H}{ }^{\frac{\alpha}{2}}(Q)}^{2} \\
& =m^{2} \cos \left(\frac{\alpha}{2} \pi\right)\left\|{ }^{C} D_{t}^{\frac{\alpha}{2}} v\right\|_{L^{2}(Q)}^{2} .
\end{aligned}
$$

Hence, we get

$$
\begin{aligned}
& m^{2} \cos \left(\frac{\alpha}{2} \pi\right)\left\|{ }^{C} D_{t}^{\frac{\alpha}{2}} v\right\|_{L^{2}(Q)}^{2}+ a_{0} \int_{Q}\left(\frac{\partial v(x, t)}{\partial x}\right)^{2} d x d t+b_{0} \int_{Q} v^{2}(x, t) d x d t \\
& \leq \int_{Q}{ }^{C} D_{t}^{\alpha} v(x, t) \cdot v(x, t) d x d t+\int_{Q} a(x, t)\left(\frac{\partial v(x, t)}{\partial x}\right)^{2} d x d t \\
&+\int_{Q} b(x, t) v^{2}(x, t) d x d t \\
& \leq \frac{1}{2 \varepsilon} \int_{Q}|f(x, t)|^{2} d x d t+\frac{\varepsilon}{2} \int_{Q}|v(x, t)|^{2} d x d t .
\end{aligned}
$$

So, we obtain

$$
\begin{array}{r}
m^{2} \cos \left(\frac{\alpha}{2} \pi\right)\left\|{ }^{C} D_{t}^{\frac{\alpha}{2}} v\right\|_{L^{2}(Q)}^{2}+\int_{Q} a_{0}\left(\frac{\partial v(x, t)}{\partial x}\right)^{2} d x d t+\int_{Q}\left(b_{0}-\frac{\varepsilon}{2}\right) v^{2}(x, t) d x d t \\
\leq \frac{1}{2 \varepsilon} \int_{Q}|f(x, t)|^{2} d x d t .
\end{array}
$$

As all the temes are positive, we have

$$
\begin{aligned}
\left\|{ }^{C} D_{t}^{\frac{\alpha}{2}} v\right\|_{L^{2}(Q)}^{2}+\left\|\frac{\partial v}{\partial x}\right\|_{L^{2}(Q)}^{2}+ & \|v\|_{L^{2}(Q)}^{2} \\
& \leq \frac{1}{2 \varepsilon}\left(\frac{1}{\min \left\{a_{0}, m^{2} \cos \left(\frac{\alpha}{2} \pi\right),\left(b_{0}-\frac{\varepsilon}{2}\right)\right\}}\right)\|f\|_{L^{2}(Q)}^{2} .
\end{aligned}
$$

Finally, it follows that

$$
\left\|{ }^{C} D_{t}^{\frac{\alpha}{2}} v\right\|_{L^{2}(Q)}^{2}+\left\|\frac{\partial v}{\partial x}\right\|_{L^{2}(Q)}^{2}+\|v\|_{L^{2}(Q)}^{2} \leq C\|f\|_{L^{2}(Q)}^{2},
$$

with

$$
C=\frac{1}{2 \varepsilon}\left(\frac{1}{\min \left\{a_{0}, m^{2} \cos \left(\frac{\alpha}{2} \pi\right),\left(b_{0}-\frac{\varepsilon}{2}\right)\right\}}\right) .
$$

Therefore, we obtain that

$$
\|v\|_{B} \leq k\|L v\|_{F}, \quad \text { where } k=\sqrt{C} .
$$

Hence the uniqueness of the solution.

Remark 4.2. This inequality $\|v\|_{B} \leq k\|L v\|_{F}$ is gives the uniqueness of the solution, indeed.

Let $v_{1}$ and $v_{2}$ two solutions, so

$$
\left\{\begin{array}{l}
L v_{1}=\mathcal{F} \\
L v_{2}=\mathcal{F}
\end{array} \Longrightarrow L\left(v_{1}-v_{2}\right)=0\right.
$$


then

$$
\left\|v_{1}-v_{2}\right\|_{B} \leq k\|0\|_{F} \Longrightarrow\left\|v_{1}-v_{2}\right\|_{B} \leq 0 \Longrightarrow v_{1}-v_{2}=0
$$

which gives the uniqueness of the solution.

Proposition 4.3. The operator $L$ from $B$ to $F$ admits a closure.

Proof. Let $\left(v_{n}\right)_{n \in \mathbb{N}} \subset D(L)$ a sequence such that

$$
v_{n} \rightarrow 0 \text { in } B
$$

and

$$
L v_{n} \rightarrow \mathcal{F} \text { dans } F
$$

it must be shown that

$$
f \equiv 0 \text {. }
$$

The convergence of $v_{n}$ toward 0 in $B$ entails that

$$
v_{n} \rightarrow 0 \text { in }\left(C_{0}^{\infty}(Q)\right)^{\prime} .
$$

As the continuity of the fractional derivation and the derivation of the first order (as a particular case of the fractional derivative) of $\left(C_{0}^{\infty}(Q)\right)^{\prime}$ in $\left(C_{0}^{\infty}(Q)\right)^{\prime}$, then (4.8) implies

$$
\mathcal{L} u_{n} \rightarrow 0 \text { in }\left(C_{0}^{\infty}(Q)\right)^{\prime} .
$$

On the other hand the convergence of $\mathcal{L} v_{n}$ to $f$ in $F=L^{2}(Q)$ implies that

$$
\mathcal{L} u_{n} \rightarrow f \text { in }\left(C_{0}^{\infty}(Q)\right)^{\prime} .
$$

By virtue of the uniqueness of the limit in $\left(C_{0}^{\infty}(Q)\right)^{\prime}$, we conclude from $(4.9)$ and $(4.10)$ that

$$
f \equiv 0 \text {. }
$$

Hence, the operator $L$ is closable.

Definition 4.4. Let $\bar{L}$ the closure of $L$ and $D(\bar{L})$ the definition domain of $\bar{L}$. The solution of the equation

$$
\bar{L} v=\mathcal{F}
$$

is called generalized strong solution of the problem $(3.1)-(3.3)$.

Theorem 1 is valid for a generalized strong solution, ie we have the following inequality:

$$
\|v\|_{B} \leq k\|\bar{L} v\|_{F}, \quad \forall v \in D(\bar{L}) .
$$

Consequently, this last inequality entails the following corollaries:

Corollary 4.5. The strong solution of the problem (3.1) - (3.3) is unique, if it exists and depends continuously on $f \in F$.

Corollary 4.6. The range $R(\bar{L})$ of the operator $\bar{L}$ is equal to the closure $\overline{R(L)}$ of $R(L)$.

Proof. Let $z \in \overline{R(L)}$, then there exists a Cauchy sequence $\left(z_{n}\right)_{n}$ in $F$ consists of the elements of the set $R(L)$ such that

$$
\lim _{n \rightarrow+\infty} z_{n}=z
$$

So there is a corresponding sequence $\left(v_{n}\right)_{n} \subset D(L)$ such that

$$
L v_{n}=z_{n} \text {. }
$$

From the estimate (4.2), we obtain

$$
\left\|v_{p}-v_{q}\right\|_{B} \leq k\left\|L v_{p}-L v_{q}\right\|_{F} \rightarrow 0, \text { lorsque } p, q \rightarrow+\infty \text {. }
$$


We can deduce that $\left(v_{n}\right)_{n}$ is a Cauchy sequence in $B$, so there is $v \in B$ :

$$
\lim _{n \rightarrow+\infty} v_{n}=v \text { in } B \text {. }
$$

By virtue of the denition of $\bar{L}\left(\lim _{n \rightarrow+\infty} v_{n}=v\right.$ in $B$; if $\lim _{n \rightarrow+\infty} L v_{n}=\lim _{n \rightarrow+\infty} z_{n}=$ $z$, so $\lim _{n \rightarrow+\infty} \bar{L} v_{n}=z$ and as $\bar{L}$ is closed so $\bar{L} v=z$ ), the function $v$ verify that

$$
v \in D(\bar{L}), \quad \bar{L} v=z \text {. }
$$

thus $z \in R(\bar{L})$, then

$$
\overline{R(L)} \subset R(\bar{L}) .
$$

So we conclude here that $R(\bar{L})$ is closed because it is complete (any complete subspace of a metric space (not necessarily complete) is closed).

It remains to show the opposite inclusion.

Let $z \in R(\bar{L})$, then there is a sequence of $\left(z_{n}\right)_{n}$ in $F$ consists of the elements of the set $R(\bar{L})$ such that

$$
\lim _{n \rightarrow+\infty} z_{n}=z .
$$

where $z \in R(\bar{L})$, because $R(\bar{L})$ is closed subset of a complete space $F$, then $R(\bar{L})$ is complete.

So there is a corresponding sequence $\left(v_{n}\right)_{n} \subset D(\bar{L})$ such that

$$
\bar{L} v_{n}=z_{n} \text {. }
$$

From the estimate (4.11), we obtain

$$
\left\|v_{p}-v_{q}\right\|_{B} \leq k\left\|\bar{L} v_{p}-\bar{L} v_{q}\right\|_{F} \rightarrow 0, \text { if } p, q \rightarrow+\infty .
$$

We can deduce that $\left(v_{n}\right)_{n}$ is a Cauchy sequence in $B$, so there is $v \in B$ :

$$
\lim _{n \rightarrow+\infty} v_{n}=v \text { in } B \text {. }
$$

Once more, there is a corresponding sequence $\left(L\left(v_{n}\right)\right)_{n} \in R(L)$ such that

$$
L v_{n}=\bar{L} v_{n} \text { over } R(L), \forall n \text {. }
$$

Then

$$
\lim _{n \rightarrow+\infty} L v_{n}=z,
$$

Consequently $z \in \overline{R(L)}$, and then we conclude that

$$
R(\bar{L}) \subset \overline{R(L)} .
$$

\section{Existence of SOLUTiON}

To show the existance of solutions, we prove that $R(L)$ is dense in $F$ for all $u \in B$ and for arbitrary $\mathcal{F}=(f, 0) \in F$.

Theorem 5.1. For $w \in L^{2}(Q)$ and for all $v \in B$, we have

$$
\int_{Q} \mathcal{L} v \cdot w d x d t=0,
$$

then $w$ vanishes almost everywhere in $Q$, this implies that the problem (3.1)-(3.3) admits a unique solution. 
Proof. The idea of the proof of the theorem is choose $w \in R(L)^{\perp}$ (exactly $w \in R(L)^{\perp} \subset$ $\left.L^{2}(Q)\right)$ and for all $v \in B$, we have and demonstrate that $R(L)^{\perp}=\{0\}$ which give $\overline{R(L)}=F$.

The scalar product of $F$ is defined by

$$
(L v, W)_{F}=\int_{Q} \mathcal{L} v \cdot w d x d t, \quad \text { where } \quad W=(w, 0) \in D(L) .
$$

The equality (5.1) can be written as follows:

$$
\int_{Q}\left({ }^{C} D_{t}^{\alpha} v(x, t)-\frac{\partial}{\partial x}\left(a(x, t) \frac{\partial v(x, t)}{\partial x}\right)+b(x, t) v(x, t)\right) \cdot w(x, t) d x d t=0
$$

where ${ }^{C} D_{t}^{\alpha} v, \frac{\partial v}{\partial x}, v \in L^{2}(Q)$, with $v$ satisfies the boundary conditions of (3.3). From (5.3), we get the equality

$$
\int_{Q}\left[{ }^{C} D_{t}^{\alpha} v(x, t) \cdot w(x, t)-\frac{\partial}{\partial x}\left(a(x, t) \frac{\partial v(x, t)}{\partial x}\right) \cdot w(x, t)+b(x, t) v(x, t) \cdot w(x, t)\right] d x d t=0
$$

And from the equality (5.4), we give the function $w$ in terms of $v$ as follows:

$$
w=v, w(x, 0)=0
$$

then $w \in L^{2}(Q)$.

Replacing $w$ in (5.4) by its representation (5.5) and integrating by parts each term of (5.4) and by taking the condition of $v$, we obtain

$$
\int_{Q}{ }^{C} D_{t}^{\alpha} v(x, t) \cdot v(x, t) d x d t+\int_{Q} b(x, t) v^{2}(x, t) d x d t+\int_{Q} a(x, t)\left(\frac{\partial v(x, t)}{\partial x}\right)^{2} d x d t=0 .
$$

According to Lemma 1,2,3 and definition 3, follow the same steps in the previous section, we have that (5.6) becomes

$$
\begin{aligned}
m^{2} \cos \left(\frac{\alpha}{2} \pi\right) \int_{Q}\left({ }^{C} D_{t}^{\frac{\alpha}{2}} v(x, t)\right)^{2} d x d t & \\
& +\int_{Q} b(x, t) v^{2}(x, t) d x d t+\int_{Q} a(x, t)\left(\frac{\partial v(x, t)}{\partial x}\right)^{2} d x d t \leq 0 .
\end{aligned}
$$

So, we obtian

$$
\begin{aligned}
m^{2} \cos \left(\frac{\alpha}{2} \pi\right)\left\|{ }^{C} D_{t}^{\frac{\alpha}{2}} v\right\|_{L^{2}(Q)}^{2} & +\int_{Q} b(x, t) v^{2}(x, t) d x d t \\
& \leq-\int_{Q} a(x, t)\left(\frac{\partial v(x, t)}{\partial x}\right)^{2} d x d t \\
& \leq-a_{0} \int_{Q}\left(\frac{\partial v(x, t)}{\partial x}\right)^{2} d x d t \\
& \leq 0,
\end{aligned}
$$

then

Hence

$$
m^{2} \cos \left(\frac{\alpha}{2} \pi\right)\left\|{ }^{C} D_{t}^{\frac{\alpha}{2}} v\right\|_{L^{2}(Q)}^{2}+b_{0}\|v\|_{L^{2}(Q)}^{2} \leq 0,
$$

$$
\|v\|_{L^{2}(Q)}=0
$$

And thus $v=0$ in $Q$ which gives $w=0$ in $Q$. So $R(L)^{\perp}=\{0\}$. This proves Theorem 2 . So $\overline{R(L)}=F$. 


\section{REFERENCES}

[1] Oldham, KB, Spanier, J: The Fractional Calculus. Academic Press, New York (1974).

[2] Kilbas, AA, Srivastava, HM, Trujillo, JJ: Theory and Applications of Fractional Differential Equations. Elsevier, Amsterdam (2006)

[3] He, JH: Nonlinear oscillation with fractional derivative and its applications. In: International Conference on Vibrating Engineering'98, Dalian, China, pp. 288-291 (1998)

[4] He, JH: Some applications of nonlinear fractional differential equations and their approximations. Bull Sci Technol15, 86-90 (1999)

[5] He, JH: Approximate analytical solution for seepage flow with fractional derivatives in porous media. Comput Methods Appl Mech Eng167, 57-68 (1998)

[6] Metzler, R, Klafter, J: The random walk's guide to anomalous diffusion: a fractional dynamics approach. Phys Rep339, 1-77 (2000)

[7] A.A. Kilbas, H.M. Srivastava, J.J. Trujillo, Theory and Applications of Fractional Differential Equations, Elsevier, Amsterdam, 2006.

[8] K.S. Miller, B. Ross, An Introduction to the Fractional Calculus and Differential Equations, J. Wiley, New York, 1993.

[9] S.G. Samko, A.A. Kilbas, O.I. Marichev, Fractional Integrals and Derivatives. Theory and Applications, Gordon and Breach, Yverdon, 1993.

[10] R.P. Agarwal, M. Benchohra, S. Hamani, Boundary value problems for fractional differential equations, Adv. Stud. Contemp. Math. 16 (2008) 181-196.

[11] A. Anguraj, P. Karthikeyan, Existence of solutions for fractional semilinear evolution boundary value problem, Commun. Appl. Anal. 14 (2010) 505-514.

[12] M. Belmekki, M. Benchohra, L. Gorniewicz, Semilinear functional differential equations with fractional order and infinite delay, Fixed Point Theory 9 (2008) 423-439.

[13] M. Belmekki, M. Benchohra, Existence results for fractional order semilinear functional differential equations, Proc. A. Razmadze Math. Inst. 146 (2008) 9-20.

[14] M. Benchohra, J.R. Graef, S. Hamani, Existence results for boundary value problems with nonlinear fractional differential equations, Appl. Anal. 87 (2008) 851-863.

[15] M. Benchohra, S. Hamani, S.K. Ntouyas, Boundary value problems for differential equations with fractional order, Surv. Math. Appl. 3 (2008) 1-12.

[16] M. Benchohra, J. Henderson, S.K. Ntouyas, A. Ouahab, Existence results for fractional order functional differential equations with infinite delay, J. Math. Anal. Appl. 338 (2008) 1340-1350.

[17] V. Daftardar-Gejji, H. Jafari, Boundary value problems for fractional diffusion-wave equation, Aust. J. Math. Anal. Appl. 3 (2006) 1-8.

[18] D. Delbosco, L. Rodino, Existence and uniqueness for a nonlinear fractional differential equation, J. Math. Anal. Appl. 204 (1996) 609-625.

[19] K. Diethelm, N.J. Ford, Analysis of fractional differential equations, J. Math. Anal. Appl. 265 (2002) $229-248$.

[20] K. Diethelm, G. Walz, Numerical solution of fractional order differential equations by extrapolation, Numer. Algorithms 16 (1997) 231-253.

[21] A.M.A. El-Sayed, Fractional order evolution equations, J. Fract. Calc. 7 (1995) 89-100.

[22] A.M.A. El-Sayed, Fractional order diffusion-wave equations, Internat. J. Theoret. Phys. 35 (2) (1996) 311-322.

[23] A.M.A. El-Sayed, Nonlinear functional differential equations of arbitrary orders, Nonlinear Anal. Theory Methods Appl. 33 (1998) 181-186.

[24] K.M. Furati, N. Tatar, Behavior of solutions for a weighted Cauchy-type fractional differential problem, J. Fract. Calc. 28 (2005) 23-42.

[25] K.M. Furati, N. Tatar, An existence result for a nonlocal fractional differential problem, J. Fract. Calc. 26 (2004) 43-51.

[26] E.R. Kaufmann, E. Mboumi, Positive solutions of a boundary value problem for a nonlinear fractional differential equation, Electron. J. Qual. Theory Differ. Equ. 3 (2007) 1-11.

[27] A.A. Kilbas, S.A. Marzan, Nonlinear differential equations with the Caputo fractional derivative in the space of continuously differentiable functions, Differ. Equ. 41 (1) (2005) 84-89.

[28] S.M. Momani, S.B. Hadid, Some comparison results for integro-fractional differential inequalities, J. Fract. Calc. 24 (2003) 37-44.

[29] S.M. Momani, S.B. Hadid, Z.M. Alawenh, Some analytical properties of solutions of differential equations of noninteger order, Int. J. Math. Math. Sci. 13 (2004) 697-701.

[30] I. Podlubny, I. Petraš, B.M. Vinagre, P. O'Leary, L. Dorcak, Analogue realizations of fractional-order controllers. Fractional order calculus and its applications, Nonlinear Dynam. 29 (2002) 281-296.

[31] C. Yu, G. Gao, Existence of fractional differential equations, J. Math. Anal. Appl. 310 (1) (2005) $26-29$. 
[32] T. Oussaeif, A. Bouziani, Existence and uniqueness of solutions to parabolic fractional differential equations with integral conditions, Vol. 2014 (2014), No. 179, pp. 1-10.

[33] D. Baleanu, J.A. Tenreiro Machado, Z.B. Guvenc, New Trends in Nanotechnology and Fractional Calculus Applications, Springer-Verlag, London, 2010.

[34] J. Sabatier, Om P. Agrawal, J.A. Tenreiro Machado, Z.B. Guvenc, Advances in Fractional Calculus: Theoretical Developments and Applications in Physics and Engineering, Springer-Verlag, London, 2007.

[35] J. Tenreiro Machado, V. Kiryakova, F. Mainardi, Recent history of fractional calculus, Commun. Nonlinear Sci. Numer. Simul. 16 (2011) 1140-1153.

[36] X. J. Li and C. J. Xu, "Existence and uniqueness of the weak solution of the space-time fractional diffusion equation and a spectral method approximation," Communications in Computational Physics, vol.8, no.5, pp.1016-1051, 2010.

[37] X. J. Li and C. J. Xu, "A space-time spectral method for the time fractional diffusion equation,"SIAM Journal on Numerical Analysis, vol.47, no.3, pp. 2108-2131, 2009.

Current address: ${ }^{1}$ Department of Mathematics and Informatics. The Larbi Ben M'hidi University, Oum El Bouaghi., ${ }^{2}$ Dynamic and control systems laboratory; Oum El Bouaghi University

BenaOuA Antara: antarabenben@gmail.com

Current address: Department of Mathematics and Informatics. The Larbi Ben M'hidi University, Oum El Bouaghi.

OUSSAEIF TAKI-EDDINE: taki_maths@live.fr; takieddine.oussaeif@gmail.com

Current address: Department of Mathematics and Informatics. The Larbi Ben M'hidi University, Oum El Bouaghi.

Rezzoug ImaD: imadrezzoug@gmail.com 